\title{
SUMOylation of the transcription factor NFATcl leads to its subnuclear relocalization and IL2 repression by HDAC
}

\author{
A Nayak*1, J Glöckner-Pagel ${ }^{1}$, M Vaeth ${ }^{1}$, M Buttmann ${ }^{2}$, T Bopp ${ }^{3}$, E Schmitt ${ }^{3}$, \\ E Serfling ${ }^{1}$ and F Berberich-Siebelt ${ }^{1}$
}

Address: ${ }^{1}$ Department of Molecular Pathology, Institute of Pathology, University of Würzburg, Würzburg, Germany, ${ }^{2}$ Department of Neurology, University of Würzburg, Würzburg, Germany and ${ }^{3}$ Institute of Immunology, University Mainz, Mainz, Germany

* Corresponding author

from 12th Joint Meeting of the Signal Transduction Society (STS). Signal Transduction: Receptors, Mediators and Genes

Weimar, Germany. 29-31 October 2008

Published: 26 February 2009

Cell Communication and Signaling 2009, 7(Suppl I):A7 doi:10.1 I86/I478-8IIX-7-SI-A7

This abstract is available from: http://www.biosignaling.com/content/7/SI/A7

(c) 2009 Nayak et al; licensee BioMed Central Ltd.

The family of NFAT (Nuclear Factor of Activated T-cells) transcription factors plays an important role in cytokine gene regulation. In peripheral T-cells, NFATc1 and c2 are pre-dominantly expressed. Due to different promoter and polyA site usage as well as alternative splicing events, NFATc1 is synthesized in multiple isoforms. The highly inducible NF-ATc1/A contains a relatively short C-terminus whereas the longer, constitutively expressed isoform NFATc1/C spans an extra C-terminal peptide of 246 amino acids. Interestingly, this NFATc1/C-specific terminus can be highly sumoylated. Upon sumoylation, NFATc1/C - but not the unsumoylated NFATc1/A - translocates to Promyelocytic Leukemia-nuclear bodies (PMLnbs). This leads to interaction with HDACs followed by deacetylation of histones, which in turn induces transcriptionally inactive chromatin. As a consequence, expression of the NFATc1 target gene interleukin-2 is suppressed. These findings demonstrate that the modification by SUMO converts NFATc1 from an activator to a site-specific transcriptional repressor, revealing a novel regulatory mechanism for NFATc1 function. 